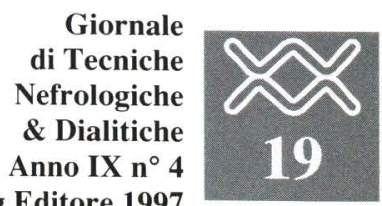

\title{
Le alterazioni del metabolismo lipidico nell'uremia: dalle fasi precoci della insufficienza renale cronica alla dialisi
}

\author{
F. Bergesio ${ }^{1}$, G. Monzani ${ }^{2}$, A.M. Ciciani ${ }^{2}$, R. Ciuti ${ }^{3}$, A. Serruto ${ }^{3}$, A. Rosati' ${ }^{1}$ R. Piperno ${ }^{1}$, \\ P.L. Tosi ${ }^{1}$, S. Bandini ${ }^{1}$, M. Salvadori ${ }^{1}$
}

\author{
${ }^{1}$ U.O. di Nefrologia e Dialisi, Ospedale di Careggi, Firenze \\ ${ }^{2}$ U.O. di Nefrologia e Dialisi, \\ ${ }^{3}$ U.O. di Chimica Clinica, Azienda USL 10 di Firenze
}

insufficienza renale cronica (IRC) si associa frequentemente ad alcune alterazioni del metabolismo lipidico come è stato ben documentato sia in patologia umana che sperimentale (1-3). Comunque il significato clinico di queste alterazioni non è ancora del tutto chiarito. Recentemente numerosi modelli sperimentali hanno dimostrato che le alterazioni del metabolismo lipoproteico possono determinare lo sviluppo di lesioni glomerulari e contribuire direttamente alla progressione della IRC $(4,5)$. Questo potrebbe portare, nella pratica clinica, all'impiego di farmaci ipolipemizzanti nel tentativo di rallentare l'evoluzione di alcune forme di malattia renale cronica (6). Un altro ruolo patogenetico, da sempre ben più conosciuto, dell'iperlipoproteinemia in corso di uremia è quello di rappresentare un importante fattore di rischio per lo sviluppo di malattia vascolare aterosclerotica $(2$, 7). Inoltre recentemente è stato suggerito che le apoproteine, alterate fin dalle fasi precoci dell'IRC, quando cioè le alterazioni lipidiche non sono ancora evidenti (8-10), possano rappresentare un indice predittivo di rischio vascolare per la malattia coronarica più preciso dei lipidi stessi $(11,12)$.

Le alterazioni del metabolismo lipoproteico che si verificano nel corso della progressione della IRC sembrano persi- stere anche durante il trattamento dialitico e dopo il trapianto renale conseguentemente al grado di recupero della funzione renale e talora aggravate dalla terapia immunosoppressiva $(1,2)$. A tutt'oggi sono pochi gli studi che abbiano esaminato le alterazioni del profilo lipidico e apoproteico di pazienti con IRC di vario grado confrontandole con quelle di pazienti in terapia dialitica periodica $(8,10.13)$. Abbiamo pertanto intrapreso questo studio ponendoci diversi obbiettivi: 1) valutare il profilo lipidico e apoproteico dei pazienti con IRC e di quelli in terapia dialitica, 2) studiare il ruolo del PTH e dell'insulina nella dislipidemia uremica e 3) determinare se le alterazioni del profilo lipidico e apoproteico riscontrate nei pazienti con IRC persistano o si modifichino nei pazienti in emodialisi (ED).

\section{Pazienti e metodi}

\section{Pazienti con IRC}

Sono stati studiati 72 pazienti (37 maschi e 35 femmine) di età media $57 \pm 10$ anni (range 35-70) affetti da IRC di vario grado e di varia eziologia. I pazienti sono stati suddivisi in due gruppi in base al valore della Clearance della Creatinina (C-Cr) corretta x $1.73 \mathrm{mq} /$ superficie corporea: il primo gruppo (IRC-I) $\mathrm{C}-\mathrm{Cr}$ $60-31 \mathrm{ml} / \mathrm{min}$ ed il secondo gruppo (IRC-II) $\mathrm{C}-\mathrm{Cr}<30 \mathrm{ml} / \mathrm{min}$. Tali gruppi sono indicati anche con i termini di IRC moderata e grave. Tutti i pazienti seguivano un regime dietetico a basso contenuto proteico (0.4-0.6 g di protidi $/ \mathrm{kg} / \mathrm{die})$ con un normale apporto calorico (30-35 $\mathrm{Kcal} / \mathrm{kg} / \mathrm{die})$.

\section{Pazienti in emodialisi}

Sono stati esaminati 31 pazienti (17 maschi e 14 femmine) di età media $54 \pm 12$ anni in trattamento dialitico periodico (IRC-ED) da un tempo medio di $77 \pm 39$ mesi. La terapia dialitica prevedeva tre dialisi alla settimana, con bagno bicarbonato o acetato single pass (senza glucosio) per la durata di 3-4 ore ogni seduta con membrane in Cuprophane, PAN o Polisulfone indifferentemente di tipo capillare o a piastra.

\section{Controlli}

28 soggetti ( 14 maschi e 14 femmine) di età media $52 \pm 9$ anni sono stati reclutati come controlli tra volontari apparentemente sani e pazienti ambulatoriali con $\mathrm{C}-\mathrm{Cr}>60 \mathrm{ml} / \mathrm{min}$.

Per tutti i gruppi, sono stati esclusi pazienti affetti da diabete mellito, abuso di alcoolici, disfunzioni tiroidee e quelli 
TABELLA I - CARATTERISTICHE CLINICHE DEI PAZIENTI E DEI CONTROLLI

\begin{tabular}{lcccc}
\hline & IRC-I & IRC-II & IRC-ED & Controlli \\
\hline Numero di soggetti & 26 & 46 & 31 & 28 \\
C-Cr (ml/min X 1.73/s.c.) & $60-31$ & $<31$ & - & $>60$ \\
C-Cr (media \pm SD) & $(41 \pm 7)$ & $(16 \pm 8)$ & - & $(92 \pm 23)$ \\
Età (anni) & $57 \pm 10$ & $57 \pm 10$ & $54 \pm 12$ & $52 \pm 9$ \\
Sesso (M/F) & $13 / 13$ & $24 / 22$ & $17 / 14$ & $14 / 14$ \\
IMC & $24 \pm 2$ & $23 \pm 3$ & $22 \pm 3$ & $24 \pm 3$ \\
$\begin{array}{l}\text { Dieta (SI*/NO) } \\
\text { Ipertensione } \\
\text { (nu. di pazienti e \%) }\end{array}$ & $13 / 13$ & $34 / 18$ & $0 / 31$ & $0 / 28$ \\
Terapia con beta-bloccanti & $15(57 \%)$ & $31(67 \%)$ & $4(13 \%)$ & $6(21 \%)$ \\
(nu. di pazienti e \%) & $2(8 \%)$ & $11(24 \%)$ & $2(6 \%)$ & $1(3 \%)$ \\
\hline
\end{tabular}

* Dieta ipoproteica convenzionale: $0.4-0.6 \mathrm{~g} / \mathrm{kg} / \mathrm{die}$

che assumevano farmaci steroidei, contraccettivi o ipolipemizzanti. Nessuna selezione è invece stata fatta per i valori di lipidi o per l'anamnesi di eventuali patologie cardiovascolari. Le principali caratteristiche dei pazienti e dei controlli sono riportate nella Tabella I.

\section{Analisi dei lipidi e delle apolipoproteine}

I campioni di sangue sono stati prelevati a digiuno in provette vacutainer contenenti EDTA $(1 \mathrm{mg} / \mathrm{ml})$, mediante prelievo venoso o dalla fistola artero-venosa prima della somministrazione di eparina per i pazienti dializzati. Dopo una centrifugazione a bassa velocità a $4{ }^{\circ} \mathrm{C}$, è stata aggiunta sodio azide alla concentrazione finale di $0.5 \mathrm{~g} / \mathrm{l}$ e analizzati subito o conservati a $-20{ }^{\circ} \mathrm{C}$; nel secondo caso i campioni sono stati analizzati tutti insieme per minimizzare le variazioni dovute all'intervallo di tempo íntercorrente tra $\mathrm{i}$ dosaggi. Il Colesterolo Totale (CT; CHOD-PAP) ed i Trigliceridi (TG) sono stati dosati sul plasma intero e le frazioni mediante il metodo enzimatico (Hoffmann/La Roche, Basilea, Svizzera). Il C-HDL è stato determinato sul sovranatante dopo precipitazione delle VLDL e LDL con una soluzione tampone di polietilenglicole (PEG 6000; concentrazione finale $10 \%$ ) a pH 10 (14).

Il C-LDL è stato calcolato dalla differenza tra Colesterolo non-LDL ottenuto dopo precipitazione selettiva delle LDL con sodio citrato (64 mmol/l, pH 5.04) e soluzione eparinata $100.000 \mathrm{U} / 1(1: 10$ $\mathrm{v}: \mathrm{v})$. Il metodo della precipitazione migliorato da Wieland e Siedel (15) per la determinazione del Colesterolo LDL sembra essere preciso e accurato come dimostrato nello studio di Demacker et al (16). I valori di Colesterolo LDL con il metodo della precipitazione sono ben correlati con quelli ottenuti con il metodo della ultracentrifugazione; Demacker et al (16) hanno dato un coefficiente di r $=0.96$.

Le apolipoproteine A1 e B (apo-A e Apo-B) sono state dosate con il metodo immunonefelometrico con un analizzatore APS Beckman (USA) secondo procedure standard (17). Gli antisieri anti-Apo -A1 e anti-Apo-B della capra (Beckman, USA), Apo-CII, CIII ed E sono state determinate con metodo immunoturbidimetrico mediante analizzatore centrifugo Cobas Fara (Hoffmann-La Roche) e gli antisieri sono stati acquistati dalla Ditta Daiichi Pure Chemicals (Giappone).

La curva di calibrazione è stata ottenuta con la diluizione di sieri standard (ApoCII, CIII, E; Daiichi).

La glicemia è stata determinata con il metodo enzimatico (GOD-PAP), la Creatininemia con il metodo colorimetrico (Jaffé modificato) e la Clearance della Creatinina è stata corretta x $1.73 \mathrm{mq} / \mathrm{su}-$ perficie corporea. L'indice di massa corporea (IMC) è stato impiegato per calcolare il peso relativo. L'insulina immunoreattiva (IRI) ed il paratormone (PTH) intatto sono stati determinati sempre su prelievi eseguiti a digiuno, usando dei kits RIA forniti rispettivamente dalla Ditta Sorin (Italia) e Nichols (USA).

\section{Analisi Statistica}

Le medie e le deviazioni standard sono state calcolate secondo il metodo convenzionale. Per l'analisi delle differenze tra i pazienti e i controlli è stato impiegato il test di Wilcoxon per dati non appaiati. Il coefficiente di correlazione di Spearman (rs) è stato usato per valutare la relazione tra due variabili. La correlazione è stata considerata statisticamente significativa per $\mathrm{p}<0.05$.

\section{Risultati}

\section{Confronto tra pazienti e controlli}

Lipidi: Un aumento dei TG e una riduzione dei livelli di C-HDL erano presenti sia nel gruppo con IRC moderata (IRCI) che in quelli con IRC grave (IRC-II), ma solo nel secondo gruppo e nel gruppo dei pazienti in terapia dialitica (IRCED) raggiungeva una significatività statistica rispetto ai controlli. Il confronto tra il gruppo IRC-II ed il gruppo IRCED non era significativo. Non si sono riscontrate differenze tra i vari gruppi sia il Colesterolo Totale (CT) che quello LDL (Fig. 1).

Apolipoproteine: Le concentrazioni di Apo-A1 hanno mostrato una riduzione di entità moderata, ma significativa sia nel gruppo IRC-II che in quello IRC-ED. Le Apo-B e Apo-E non erano significativamente alterate tranne che nel gruppo dei pazienti dializzati dove si aveva un marcato aumento delle Apo-E (Fig. 2). Le Apo C-II avevano lo stesso andamento. Al contrario le Apo-C-III risultavano elevate fin dalle fasi precoci della IRC (IRC-I) ed aumentavano ulteriormente nei gruppi IRC-II e IRC-ED (Fig. 3). I rapporti Apo-C-II/Apo-C-III ed ApoE/Apo-C-III hanno mostrato una progressiva riduzione con la progressione della malattia renale, dal gruppo IRC-I al gruppo IRC-ED; in particolare il primo rapporto risultava diminuito già quando il filtrato glomerulare era $<60 \mathrm{ml} / \mathrm{min}$ mentre il secondo rapporto diminuiva solo nel gruppo IRC-II e in quello IRC-ED (Fig. 3). Inoltre i pazienti dializzati mostravano una significativa riduzione del rapporto Apo-E/Apo-C-III rispetto al gruppo con IRC grave, ma non ancora in dialisi (IRC-II). I dializzati inoltre avevano livelli significativamente più elevati di Apo-C-II, Apo C-III e Apo-E rispetto ai pazienti con IRC grave.

PTH, IRI: In tutti i gruppi sono stati riscontrati alti livelli di PTH . Comunque 
il gruppo IRC-ED mostrava una riduzione moderata ma significativa rispetto al gruppo IRC-II. Al contrario non veniva messa in evidenza nessuna differenza per i valori di IRI ad eccezione che nei pazienti in terapia dialitica che mostravano un aumento molto significativo a confronto sia dei controlli che del gruppo IRC-II (Fig.4).

\section{Analisi delle correlazioni}

Le analisi sono state eseguite considerando i pazienti con IRC moderata e grave come un unico gruppo (IRC-I + IRC-II). I TG sono risultati inversamente correlati ai livelli di HDL-C sia nei gruppi con IRC in terapia conservativa che in quello dei pazienti in terapia dialitica $(\mathrm{rs}=-0.349, \mathrm{p}=0.003$ e $\mathrm{rs}=0.533$, $\mathrm{p}=0.002)$. Inoltre i TG risultavano significativamente correlati in tutti i gruppi alle Apo-CII ( $r s=0.695, p<0.001$ e $\mathrm{rs}=0.486, \mathrm{p}<0.01)$, alle Apo-CIII ( $\mathrm{rs}=$ $0.773, \mathrm{p}<0.001$ e $\mathrm{rs}=0.629, \mathrm{p}=0.005)$ e alle Apo-E (rs $=0.546, \mathrm{p}<0.001$ e rs $=$ $0.501, \mathrm{p}=0.004)$. Al contrario una correlazione significativa tra TG ed Apo-B è stata trovata solo nel gruppo IRC-ED (rs $=0.676, p<0.001)$. Nessuna relazione è stata messa in evidenza tra $\mathrm{i}$ TG ed i rapporti Apo C-II/Apo-C-III e Apo-CII/Apo-E. Tutti i gruppi avevano una stretta correlazione tra C-HDL ed Apo$\mathrm{Al}(\mathrm{rs}=0.786, \mathrm{p}<0.01$ e rs $=0.673, \mathrm{p}<$ 0.001).

Nessuna correlazione è stata osservata tra TG e livelli di PTH in ambedue $\mathrm{i}$ gruppi (Tab. II). Al contrario i TG mostravano una correlazione altamente significativa con i livelli di IRI nei pazienti dializzati (Tab. III). Inoltre nello stesso gruppo erano significativamente correlati PTH e IRI ( Tab. II).

\section{Discussione}

I risultati dello studio mettono in evidenza una relazione tra la progressione dell'insufficienza renale e alcune specifiche alterazioni del metabolismo lipoproteico. Nelle fasi precoci della malattia (IRC-I, VFG $60-31 \mathrm{ml} / \mathrm{min}$ ) si assiste ad un aumento dei $\mathrm{TG}$ e ad una riduzione del $\mathrm{C}$ HDL. Comunque queste alterazioni raggiungono una significatività statistica solo nei pazienti con IRC grave (IRC-II, $\mathrm{VFG}<30 \mathrm{ml} / \mathrm{min}$ ) e in quelli in terapia dialitica (IRC-ED). Al contrario non ab-

\begin{tabular}{|c|c|c|c|c|}
\hline & \multicolumn{2}{|c|}{$\begin{array}{l}\text { PTH } \\
\text { IRC-I + IRC-II } \\
\quad(n=72)\end{array}$} & \multicolumn{2}{|c|}{$\begin{array}{l}\text { IRC-ED } \\
(\mathrm{n}=31)\end{array}$} \\
\hline & rs & $\mathrm{p}$ & rs & $\mathrm{p}$ \\
\hline Trigliceridi & 0.185 & NS & 0.282 & NS \\
\hline Colesterolo Totale & -0.159 & NS & -0.074 & NS \\
\hline Colesterolo - HDL & 0.242 & 0.042 & -0.489 & 0.005 \\
\hline Colesterolo - LDL & 0.218 & NS & 0.147 & NS \\
\hline Apo-A1 & -0.16 & NS & 0.203 & NS \\
\hline Apo-B & -0.213 & NS & -0.158 & NS \\
\hline Apo-C-II & -0.103 & NS & -0.038 & NS \\
\hline Apo-C-III & -0.052 & NS & -0.028 & NS \\
\hline Rapporto Apo-C-II/Apo-C-III & -0.138 & NS & -0.146 & NS \\
\hline Apo-E & 0.151 & NS & 0.18 & NS \\
\hline IRI & 0.091 & NS & 0.0372 & 0.04 \\
\hline
\end{tabular}

$\begin{aligned} \text { TABELLA III - } & \text { CORRELAZIONE STATISTICA (rs - COEFFICIENTE DI } \\ & \text { SPEARMAN) TRA IRI, LIPIDI E APOLIPOPROTEINE NEI } \\ & \text { PAZIENTI CON IRC E QUELLI IN ED }\end{aligned}$

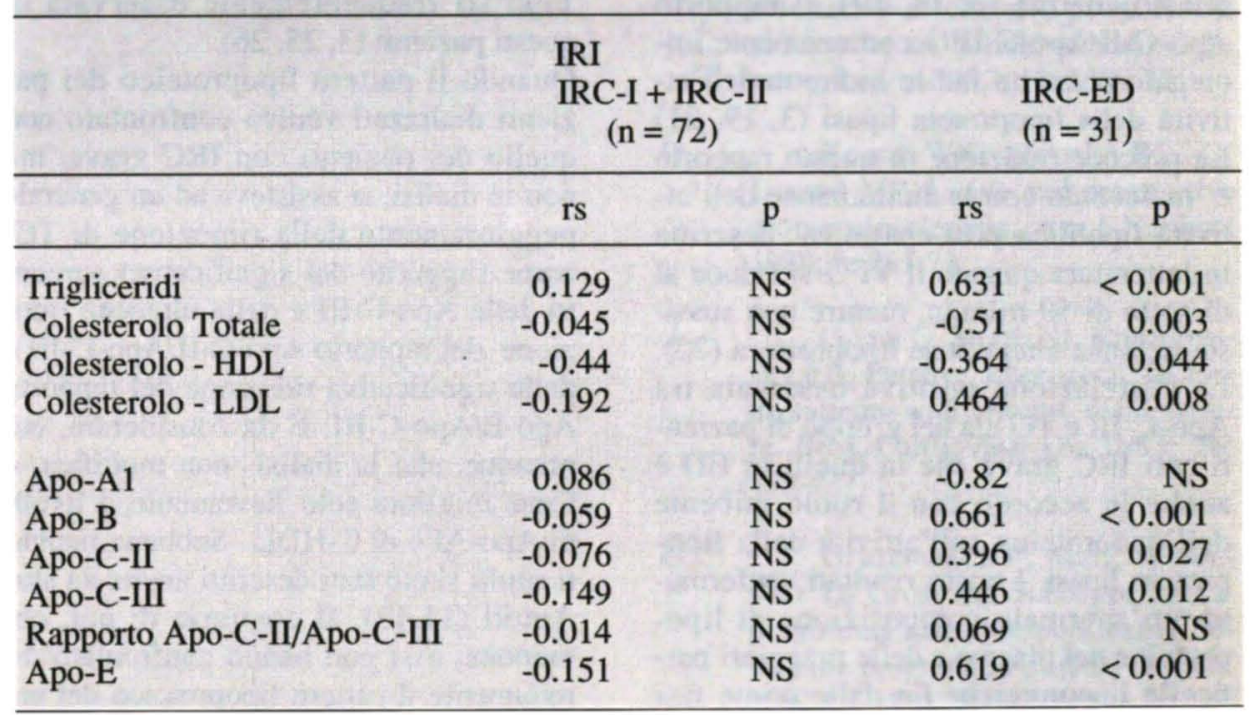

biamo trovato nessuna differenza significativa per i livelli di CT e di C-LDL. Risultati analoghi sono stati riportati da Attman e Alaupovic (9) e da Massy et al (13) in pazienti con IRC di vario grado o in terapia dialitica.

Per quanto riguarda le apolipoproteine, l'alterazione più tipica dell'uremia è rappresentata dal marcato aumento delle Apo-C-III che risulta particolarmente evidente nei pazienti con IRC avanzata ed in quelli in terapia dialitica. Inoltre, abbiamo osservato una riduzione moderata, ma significativa, dei livelli di ApoA1 nei gruppi IRC-II e IRC-ED. Gli stessi risultati sono stati riscontrati da Attman e Alaupovic (9) e da Massy et al (13) anche in pazienti con un VFG $>15$ $\mathrm{ml} / \mathrm{min} \mathrm{e}>18 \mathrm{ml} / \mathrm{min}$ rispettivamente. In accordo con questi risultati, Attman e 
Alaupovic (9) hanno riportato una riduzione dei rapporti Apo-A1/Apo-C-III, Apo-B/Apo C-III, Apo-Al/Apo-B e Apo C-III/Apo E. Analogamente Massy et al (13) ha riscontrato una significativa riduzione di Apo C-III nei pazienti con iniziale insufficienza renale.

Le alterazioni precoci delle apolipoproteine osservate da questi Autori possono essere attribuite al diverso criterio di reclutamento dei pazienti. Infatti nei loro studi il gruppo con insufficienza renale meno grave includeva pazienti con VFG $>15 \mathrm{ml} / \mathrm{min}$ e $18 \mathrm{ml} / \mathrm{min}$ rispettivamente. Al contrario il nostro gruppo IRC-I include pazienti con VFG $>30 \mathrm{ml} / \mathrm{min}$. Inoltre $\mathrm{i}$ differenti regimi dietetici, la terapia antipertensiva o altri fattori potrebbero aumentare le discrepanze con i nostri risultati.

Nel nostro studio l'insufficienza renale iniziale (C-Cr 60-31 ml/min) era caratterizzata da una isolata e significativa riduzione del rapporto Apo-C-II/Apo-C-III, non associata ad importanti alterazioni del pattern lipidico.

Dal momento che la Apo-C-II agisce come cofattore dell'attività della lipoprotein lipasi, mentre la Apo-C-III inibisce questo enzima $(3,18,19)$, il rapporto Apo-C-II/Apo-C-III è comunemente impiegato come un indice indiretto dell'attività della lipoprotein lipasi $(3,19,21)$ La precoce riduzione di questo rapporto è in accordo con la diminuzione dell'attività lipolitica post-eparinica descritta in letteratura quando il VFG si riduce al di sotto di $50 \mathrm{ml} / \mathrm{min}$, mentre non sussiste nessuna alterazione lipoproteica (22). La correlazione positiva osservata tra Apo-C-III e TG sia nel gruppo di pazienti con IRC grave che in quelli in ED è anche in accordo con il ruolo inibente dell'apoproteina sull'attività della lipoprotein lipasi. I nostri risultati confermano un'anormale composizione di lipoproteine nel plasma e delle maggiori particelle lipoproteiche fin dalle prime fasi dell'IRC (10-13). A conferma di questo, è stata trovata un'alterata concentrazione di lipoproteine anche nei soggetti uremici normolipemici $(13,23)$.

L'aumento di Apo-C-III può contribuire anche a compromettere la lipolisi riducendo l'uptake delle particelle remnant da parte del fegato: infatti si pensa che un eccesso di apoproteine inibisca la loro captazione (3). Invece la rimozione di remnant da parte del fegato è aumentata dai livelli plasmatici di Apo-E (19-24).
Quindi il rapporto Apo-E/Apo-C-III è considerato un indiretto marker della rimozione delle particelle LDL remnant dal fegato In accordo con i risultati di Attman e Alaupovic (9), i livelli di ApoE non sono alterati nella IRC in terapia conservativa, mentre mostrano un marcato aumento nei dializzati. Ma questi risultati non sono stati confermati in un recente lavoro (13). Comunque il rapporto Apo-C-II/Apo-C-III diminuisce progressivamente dalle fasi precoci della IRC fino alla dialisi, facendo pensare ad una progressiva compromissione della clearance delle particelle LDL remnant. Nonostante il marcato aumento dei livelli di Apo-E, i pazienti in dialisi mostrano una riduzione del rapporto Apo-E/ApoC-III rispetto ai pazienti con IRC grave ancora in terapia conservativa, suggerendo un ulteriore accumulo di particelle remnant $(3,25,26)$.

$\mathrm{Si}$ riscontra facilmente un aumento del rapporto Apo-C-III/Apo-E nelle VLDL e LDL dei pazienti con IRC in fase iniziale (11, 27, 28). D'altra parte, un aumento delle particelle LDL remnant è una caratteristica comune nell'uremia e si pensa che conduca alla Iperlipoproteinemia Tipo III frequentemente osservata in questi pazienti $(3,25,26)$.

Quando il pattern lipoproteico dei pazienti dializzati veniva confrontato con quello dei pazienti con IRC grave, ma non in dialisi, si assisteva ad un generale peggioramento della rimozione di $\mathrm{TG}$, come suggerito dal significativo aumento delle Apo-C-III e dalla ulteriore riduzione del rapporto Apo-C-II/Apo-C-III e dalla significativa riduzione del rapporto Apo-E/Apo-C-III. È da considerare, comunque, che la dialisi non modifica, o forse migliora solo lievemente, i livelli di Apo-Al e di C-HDL. Sebbene risultati simlli siano stati descritti anche da altri Autori $(11,13)$, al contrario di noi, comunque, essi non hanno confrontato direttamente il pattern lipoproteico dei pazienti in ED con quello dei pazienti con IRC grave.

L'assenza di una correlazione tra PTH e TG nella IRC e in ED esclude un ruolo importante di questo ormone nella patogenesi della ipertrigliceridemia uremica. Invece la correlazione significativa tra $\mathrm{i}$ TG e i livelli di insulina nel gruppo IRCED potrebbe suggerire un contributo dell'iperinsulinismo per il loro aumento. Una correlazione simile è stata riportata da Chan et al (2). L'iperinsulinismo in- fatti potrebbe stimolare la sintesi epatica di TG e quindi potrebbe contribuire in qualche modo alla dislipidemia dei pazienti in dialisi (29).

I nostri risultati dimostrano una precoce e progressiva riduzione nel rapporto Apo-C-II/Apo-C-III nella IRC, confermando il fatto che le apolipoproteine sono un marker delle alterazioni precoci del metabolismo lipidico, più sensibile dei lipidi stessi. L'aumento dei livelli di Apo-C-III e la riduzione del rapporto Apo-C-II/Apo-C-III sono le caratteristiche più tipiche della dislipidemia uremica e danno un'indiretta dimostrazione del fatto che la iperlipidemia dipende principalmente da una inadeguata rimozione di TG.

Il ridotto rapporto Apo-E/Apo-C-III è in accordo con un'ulteriore riduzione dell'attività lipolitica e con un aumento di accumulo di remnant, specialmente nei pazienti in dialisi.

In conclusione, la terapia dialitica sembra peggiorare la rimozione di TG mentre stimolerebbe la sintesi epatica di TG mediante l'aumento dei livelli di insulina. I nostri risultati dimostrerebbero d'altra parte che la dialisi non agisce su quei fattori (particolarmente il C-HDL e le Apo-B) che sono generalmente considerati importanti nello sviluppo dell'aterosclerosi (1-3). Accanto alle alterazioni delle lipoproteine riconosciute come uno dei maggiori fattori di rischio per la malattia cardiovascolare, molti altri fattori di rischio possono essere attivi nei pazienti con IRC e in quelli in ED, quali l'abitudine al fumo, l'ipertensione, le alterazioni del metabolismo calcio-fosforo, l'intolleranza glicidica e altre tossine uremiche che potrebbero essere implicate nell'ispessimento della parete vascolare. La dialisi complica ulteriormente queste alterazioni introducendo altre variabili. L'uso continuativo di eparina, per esempio, durante il trattamento dialitico inibisce la lecitin-colesterol-aciltransferasi e potrebbe esaurire il pool di lipoprotein lipasi (2).

Nel trattamento dell'iperlipoproteinemia è importante in primo luogo un adeguato regime dietetico (30.31) e una costante attività fisica. Questi provvedimenti servono per mantenere un normale peso corporeo e per ridurre la concentrazione dei lipidi nel plasma. In secondo luogo è importante eliminare altri fattori quali il diabete, l'abuso di alcool ecc.

Infine abbiamo la terapia con $\mathrm{i}$ farmaci 
che diminuiscono la concentrazione dei lipidi nel plasma sia diminuendo la produzione di lipoproteine sia aumentando la loro rimozione.

Un concetto attuale è la combinazione terapeutica di farmaci che abbiano effetti sinergici nell'abbassare la concentrazione dei lipidi nel plasma, specialmente quelli che agiscono sul C-LDL. Per inibire la sintesi di colesterolo si usano gli inibitori della HMG-CoA reduttasi (statine), mentre gli acidi fibrici (gembfibrozil, clofibrato e fenfibrato) hanno dimostrato efficacia nel trattamento sia della ipertrigliceridemia che della ipercolesterolemia $(32,33)$.

In conclusione, l'uso di farmaci ipolipemizzanti è ampiamente raccomandato per la prevenzione della malattia cardiovascolare aterosclerotica e recentemente potrebbe trovare impiego anche nella prevenzione della progressione della IRC.

\section{Riassunto}

Nell'insufficienza renale cronica (IRC) si riscontrano frequentemente alcune alterazioni del metabolismo delle lipoproteine, sia nel periodo della terapia conservativa che durante la terapia dialitica. Per studiare le variazioni del metabolismo lipidico e lipoproteico dalle fasi precoci della IRC alla dialisi, sono stati dosati i lipidi plasmatici con le apoproteine A1, B, E, C-II, C-III, i rapporti Apo-C-II/Apo-C-III ed Apo-E/Apo-CIII, il paratormone (PTH) e l'insulina in 72 pazienti con IRC di vario grado e 31 pazienti in terapia dialitica e poi sono stati confrontati con i valori di 28 controlli. L'alterazione riscontrata più precocemente è stata una significativa riduzione del rapporto Apo-C-II/Apo-C-III; mentre solo nelle fasi più avanzate della malattia (Clearance della Creatinina $<31$ $\mathrm{ml} / \mathrm{min}$ ) si metteva in evidenza un aumento dei Trigliceridi con ridotti livelli di Colesterolo HDL, un aumento di ApoC-III e riduzione del rapporto Apo E/Apo-C-III. I pazienti in terapia dialitica mostravano un peggioramento generale del profilo lipoproteico con elevate Apo-E e una indiretta evidenza di accumulo di remnants. Inoltre mentre il PTH non sembrava avere una significativa influenza sulle lipoproteine, l'aumento di insulinemia durante la terapia dialitica potrebbe avere un ruolo nella patogenesi della ipertrigliceridemia di questi pazienti. Dai nostri risultati si può concludere che le alterazioni più tipiche della dislipidemia uremica sono rappresentate dall'aumento delle Apo-C-III e quindi dalla riduzione del rapporto Apo-C-II/Apo-CIII e Apo-E/Apo-C-III e che il principale meccanismo patogenetico della ipertrigliceridemia uremica potrebbe dipendere da un difetto nella rimozione dei trigliceridi. La terapia dialitica sembra aggravare globalmente le alterazioni dei lipidi e delle lipoproteine osservati nel periodo pre-dialitico.

\section{BIBLIOGRAFIA}

1. Bagdade JD, Carretto A, Albers J. Effects of chronic uremia, hemodialysis and renal transplantation on plasma lipids and lipoproteins in man. J Lab Med 1976; 1987: 37-48.

2. Chan MK, Varghese Z, Moorhead JF. Lipids abnormalities in uremia, dialysis and transplantation. Kidney Int 1981; 9: 625-37.

3. Lacour B, Drueke TB. Lipid metabolism. In: Massry SG, Chassock RJ, eds. Textbook of Nephrology. Baltimore, Williams \& Wilkins 1989; vol. 2: 1228-38.

4. Kasiske BL, Clearly MP, Kean WF. Effects of genetic obesity on renal structure and function in the Zucker rat. J Lab Clin Med 1985; 106: 598-604.

5. Moorhead JF, El-Nahas M, Chan MK, Varghese Z. Lipid nephrotoxicity in chronic progressive glomerular and tubulo-interstitial disease. Lancet 1982; 11: 1309-11.

6.

Keane WK, Kasiske BL, O' Donnel MP. Lipids and progressive glomerulosclerosis. Am J Nephrol 1988; 8: 261-71.

7. Green D, Stone NJ, Krumlovasky FA. Putative atherogenic factors in patients with chronic renal failure. Prog Cardiovasc Dis 1983; 26: 133-44.

8. Grutzmaker P, Marz W, Peshke B, Gross W, Schoeppe L. Lipoproteins and apolipoproteins during the progression of renal disease. Nephron 1988; 50: 103-11.

9. Attman PO, Alaupovich P. Lipid and apolipoprotein profiles of uremic dyslipoproteinemia. Relation to renal function and dialysis. Nephron 1991; 54: 401-10.

10. Bergesio F, Monzani G, Ciuti R, et al. Lipids and apolipoproteins change during progression of chronic renal failure. Clin Nephrol 1992; 38: 264-70. 
11. Alaupovich P, McConathy WJ, Fasmire J, Tavella M, Mard JM. Profiles of particles in dyslipoproteinemias. Clin Chem 1988; 33: B1327.

12. Brunzell JD, Sniderman AD, Albers J, Kwiterovich PO. Apolipoproteins $\mathrm{B}$ and $\mathrm{A} 1$ and coronary $\mathrm{Ar}$ tery disease in humans. Atherosclerosis 1984; 4: 79-83.

13. Massy ZA, Jungers P, Roullet JB, Druerke T, Lacour B. Disturbances of apolipoprotein distribution in lipoproteins of uremic patients. J Nephrol 1993; 6: 153-8.

14. Izzo C, Grillo F, Murador F. Improved method for determination of high-density lipoprotein cholesterol. I. Isolation of HDL by use of polyethylene glycol 6000. Clin Chem 1981; 27: 371-4.

15. Wieland H, Seidel D. A simple specific method for precipitation of low-density lipoproteins. J Lipid Res 1983; 23: 904-9.

16. Demacker PN, Hijmans AG, Brenninkmeijr BJ, Jansen ADP, Van'T Laar A. Five methods for determining low density lipoprotein cholesterol compared. Cli Chem 1984; 30: 1797-800.

17. Weinstock N, Batholone M Determination of Apo A1 by kinetic nephelometry. Biochim Biophys Acta 1981; 663: 279-88.

18. Nilsson-Ehle P, Garfinkel AS, Schotz MC. Lipolytic enzyme and plasma lipoprotein metabolism. Rev Biochem 1980; 49: 667-93.

19. Dolphin PJ. Lipoprotein metabolism and the role of apolipoproteins as metabolic programmers. Can J Biochem Cell Biol 1985; 63: 850-69.

20. Strapans I, Felts JM, Zacherle B. Apoprotein composition of plasma lipoproteins in uremic patients on hemodialysis. Clin Chim Acta 1979; 93: 135-43.

21. Attman PO, Alaupovich P, Gustafson A. Serum apolipoprotein profile of patients with chronic renal failure. Kidney Int 1987; 32: 368-75.
22. McCosh EJ, Solangi K, Rives JM, Goodman A. Hypertriglyceridemia in chronic renal insufficiency. Am J Clin Nutr 1975; 28: 1936-43.

23. Samuelsson O, Attman PO Knight-Gibson C, et al. Lipoprotein abnormalities without hyperlipidaemia in moderate renal insufficiency. Nephrol Dial Transplant 1994; 9: 1580-5.

24. Windler E, Havel RJ. Inhibitory of triglyceride-rich lipoproteins and their remnants by perfused rat liver. Lipid Res 1985; 27: 556-65.

25. Nesterl PJ, Fidge NH, Tan $\mathrm{MH}$. Increased lipoprotein-remnant formation in chronic renal failure. $\mathrm{N}$ Engl J ed, 1982; 397: 329-33.

26. Ron D, Oren I, Aviram M, Better OS, Brook JG. Accumulation of lipoprotein remnants in patients with chronic renal failure. Atherosclerosis 1983; 46: 67-75.

27. Camejo G, Riera G, Lee M, Lopez F. Lipoprotein structural abnormalities in chronic renal failure with and without hemodialysis. Biomed Biochim Acta 1988; 4: 239-45.

28. Wakabayashi Y, Okubo M, Shimade H, Koide A, Mamumo F, Nakamura H. Decreased VLDL Apoprotein CII/Apoprotein CIII ratio may be seen in both normotriglyceridemic and hypertriglyceridemic patients on chronic dialysis treatment. Metabolism 1987; 38: 601-2.

29. Chan MK, Varghese Z, Persaud W, Baillood RA, Moorhead JF. Hyperlipidemia in patients on maintenance hemo-and peritoneal dialysis: the relative pathogenetic roles of triglycerides production and triglycerides removal. Clin Nephrol 1992; 37: 183-90.

30. Ritz E, Augustin J, Bommer J, Guasso A, Haberbosch W. Should hyperlipemia of renal failure be treated? Kidney Int 1985; 28 (Suppl): 84-7.

31. Goldberg AC, Schonfeld G. Effect of diet on lipoprotein metabolism. Ann Rev Nutr 1985; 5: 195-212
32. Grundy SM. Management of hyperlipidemia of kidney disease. Kideny Int 1990; 37: 847-53.

33. Appel G. Lipid abnormalities in renal disease. Kidney Int 1991; 39: 169-83. 